\title{
Interactive comment on "Reviews and syntheses: Gaining insights into evapotranspiration partitioning with novel isotopic monitoring methods" by Youri Rothfuss et al.
}

\section{Anonymous Referee \#2}

Received and published: 20 December 2020

The submitted manuscript reviewed the methods of measuring or estimating $\delta \mathrm{E}, \delta \mathrm{T}$ and $\delta \mathrm{ET}$ for identifying the possible challenges of these isotopic methods, and how they should progress in the future, especially novel non-destructive methods. While this study is meaningful for the use of isotopic partitioning methods, the current literature overview and methodological review did not offer enough supports for the forementioned objectives. However, before the paper can be published, a major revision for this manuscript will be needed. The general and specific comments are showed as follows: General comments: 1. Literature overview: A literature overview was listed in the section 2 as a timeline for highlighting the important progresses made over the past 30 years, however, it might be helpful to classify and summarize these literature. If pos- 
sible, suggest to add the topic sentences at the beginnings of each paragraphs for understanding easily. 2. Methodological review: Suggest adding a theoretical schematic diagram including the flux of soil evaporation (E), transpiration $(T)$, evapotranspiration $(E T)$, and their stable isotopic compositions, and the key points in the estimation of $\delta \mathrm{E}, \delta \mathrm{T}$, and $\delta \mathrm{ET}$ in an ecosystem for understanding the calculation principle clearly. 3. Methodological review: One of assumptions of Keeling plot approach is temporal variations in the water vapor mixing ratio and $\delta \mathrm{V}$ are caused only by ET. However, rainfall, entrainment process of air, and so on can inïňĆuence the variation in $\delta \mathrm{V}$ at hourly or daily time scales, and introduces biases in the Keeling plot estimates. Suggest to add the analysis of the related uncertainties for Keeling plot approach. 4. Methodological review: Suggest to add the comprehensive comparisons of Keeling plot, flux gradient and EC isotopic ïňĆux method according to the calculation principle. Keeling plot and flux gradient methods can be agreement under certain conditions, and both calculation principles of flux gradient and EC isotopic ïňĆux methods is micrometeorological theory, however the discrepancy still exist among them (eg. Hu et al., 2020 etc.). Hu, Y., Xiao, W., Wei, Z., Welp, L., Wen, X., Lee, X. (2020) Determining the Isotopic Composition of Surface Water Vapor Flux From High-Frequency Observations Using Flux-Gradient and Keeling Plot Methods. Earth and Space Science, DOI: 10.1002/essoar.10501239.1. 5. Methodological review: How about the uncertainty analysis of EC isotopic ïň́ux methods due to the loss of information during the covariance calculation between the isotopic compositions and vertical wind ïňCuctuations? 6. Methodological review: Suggest to clarifying the calculations and assumptions of converting diŻ£ value of water vapor into ðiŻ£ value of soil or xylem (liquid) water, and the analysis of possible uncertainty in detailed, for promoting the use of the novel non-destructive methods in the future. 3. Possible ways forward: It might be useful to focus on the improvement of difficult problems of recommended methods, for example, how the plant-size measurements based on the chamber methods are scaled up for canopy-level estimations. Specific comments: 1. Line 405: if the describe of "the second relies on destructive sampling of the soil and offline analysis of the extracted water" is suitable, and the non-

Printer-friendly version

Discussion paper
Interactive comment

C2 
destructive collection and online monitoring of the water vapour isotopic composition of soil atmosphere also can be used. 2. Line 640-670: Péclet effect is very important for these theoretical equations, however, its definition and quantification are not explicitly named here. 3. Line 735: For the cryogenic extraction methods of xylem water, new biases might be considered, because Chen et al. (2020) found that a dynamic exchange between organically bound deuterium and liquid water during water extraction can cause the stem water cryogenic extraction error, rather than deuterium fractionation occurs during root water uptake. Chen, Y., Helliker, B.R., Tang, X., Li, F., Zhou, Y. and Song, X. (2020) Stem water cryogenic extraction biases estimation in deuterium isotope composition of plant source water. Proceedings of the National Academy of Sciences, 202014422.

Interactive comment on Biogeosciences Discuss., https://doi.org/10.5194/bg-2020-414, 2020. 